\title{
Insurepp-Machine Learning Webapp
}

\author{
Rahul Singh, Avnesh Nigam, S. Godfrey Winster
}

\begin{abstract}
Nowadays, there are many companies which are collecting money in the name of insurance. For them, insurance has become a type of business. To reduce this thing, we have developed INSUREPP which can help in giving less amount and is very easy to use. You just need to click some pictures and upload it in the application. It will use various CNN models. It will check the harm, the seriousness of the harm, the region of the harm and will predict the results. We are making this project so that it takes less time in insurance claiming, as it can predict the cost of damage.
\end{abstract}

Keywords: INSUREPP, CNN models.

\section{INTRODUCTION}

Insurance is a technique for confirmation from money related mishap. It is a kind of peril the heads, essentially used to fence against the risk of a surprising or sketchy misfortune.An component which gives insurance is known as a back up plan, an insurance organization, an insurance carrier or an underwriter. An individual or component who buys insurance is alluded to as an ensured or as a policyholder. The insurance trade incorporates the defended hoping to be a guaranteed and referred to - tolerably little - adversity as portion to the guarantor as a trade-off for the safety net provider's assurance to reimburse the ensured in the event of a covered mishap. The incident may be financial, anyway it ought to be reducible to financial terms, and typically remembers something for which the shielded has an insurable interest set up by ownership, possession, or earlier relationship. In short, insurance is finished by an individual in order to secure something which is extremely dear to his/her life.Car is something such which is near an individual's heart.An insurance should be asserted since, supposing that some setback happens, that individual can guarantee insurance. INSUREPP can help in guaranteeing insurance. Companies take a ton of time in asserting insurance, either in weeks or in months, but INSUREPP can guarantee in only few minutes, by the outcomes from the photos that are transferred.

Manuscript received on April 20, 2021.

Revised Manuscript received on June 03, 2021.

Manuscript published on June 30, 2021.

* Correspondence Author

Rahul Singh*, Student, Department of Computer Science and Engineering, SRM Institute of Science and Technology, Chennai, India. Email: rr4872@srmist.edu.in

Avnesh Nigam, Student, Department of Computer Science and Engineering, SRM Institute of Science and Technology, Chennai, India. Email: am3010@srmist.edu.in

Dr. S. Godfrey Winster, Assistant Professor, Department of Computer Science and Engineering, SRM Institute of Science And Technology, Chennai, India. Email: godfreys@srmist.edu.in

(C) The Authors. Published by Blue Eyes Intelligence Engineering and Sciences Publication (BEIESP). This is an open access article under the CC BY-NC-ND license (http://creativecommons.org/licenses/by-nc-nd/4.0/)

\section{STATE OF THE ART (LITERATURE SURVEY)}

1. CSPNet: A New Backbone that can Enhance Learning Capability of CNN

In view of a creating availability of a great deal of data and extended computational power, data analysts have manufactured models that perform well in different PC vision endeavors

2. License plate recognition using fast RCNN and OCR: NEED, TREND, CHALLENGES AND OPPORTUNITIES

In this Project we utilize many picture division strategies alongside RCNN to distinguish tags and read its number utilizing OCR.

3. A NOVEL INFORMATION PROCESSING IN IOT BASED REAL TIME CNN photo Model and its SYSTEM

Using sensors, the condition of the individual could be dealt with as data which is aggregately named as IoT sensor organizations.

4. Neural Network-Based Vehicle and Pedestrian Detection for Video

In this work vehicles and walkers are viewed as objects of interest. Current artificial neural organizations can recognize and restrict objects of known classes.

5. Trends, Problems And Solutions of Recommender System

It utilizes a few strategies for suggestion which incorporates content-based, shared and mixture techniques.

6. Multi-Task Learning for Entity Recommendation and Document Ranking in Web Search

We study the issue of setting mindful and customized substance proposal, furthermore, propose a perform multiple tasks learning system with profound neural organizations to mutually learn two partner undertakings in web indexes: substance suggestion and report positioning, which can be effectively prepared in a start to finish way.

7. CNNpred: CNN-based stock market prediction using a diverse set of variables

In this paper, experts from the University of Tehran present their CNN-based framework, CNNpred, for remembering extraction and perceptive examination for protections trade data.

8. The Lottery Ticket Hypothesis: Finding Sparse, Trainable Neural Networks

It uses algorithm to recognize winning tickets and a movement of preliminaries that help the lottery ticket hypothesis and the meaning of these irregular presentations.

Published By: Blue Eyes Intelligence Engineering and Sciences Publication

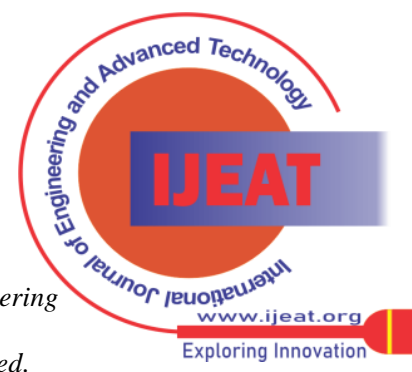


9. Introduction to CNN Keras - 0.997

It fills in as a novice instructional exercise on the best way to assemble a 5 layer $\mathrm{CNN}$ for digit acknowledgment. Worked with Keras API, this inside and out instructional exercise goes through each progression from information planning to show assessment.

10. Mask R-CNN

One of the best assessed CNN papers on Papers With Code, Mask R-CNN achieved a SOTA (bleeding edge) rating for the Instance Segmentation on Cityscapes test. In this paper, the Facebook AI Research bunch presents a clear, yet versatile framework for object model division.

\section{MAIN REASON FOR USING MACHINE LEARNING}

1. Companies are advancing-Nowadays,companies are using some new machine learning methods to run the services like healthcare,customer services,self driven cars,etc.

2. Sources can be found everywhere- Nowadays, the sources are available everywhere,and so the open source protocols can help in sharing and using the data,as it can be helpful to others.In future,some companies can help in making data sharing private using common regulatory and using cybersecurity.

3. Harnessing IOT data- Natural-language processing algorithms are ceaselessly progressing. Man-made intelligence is getting capable at understanding communicated in language and at facial acknowledgment, assisting with making it more valuable and instinctive. These algorithms are advancing startlingly, as Google found when Google Translate created its own language to assist it with deciphering effectively.It represents the development of the $\mathrm{AI} / \mathrm{AI}$ market in various geological locales more than 10 years. Fig-1 shows the speeding up reception of AI and the basic significance of this innovation pattern.

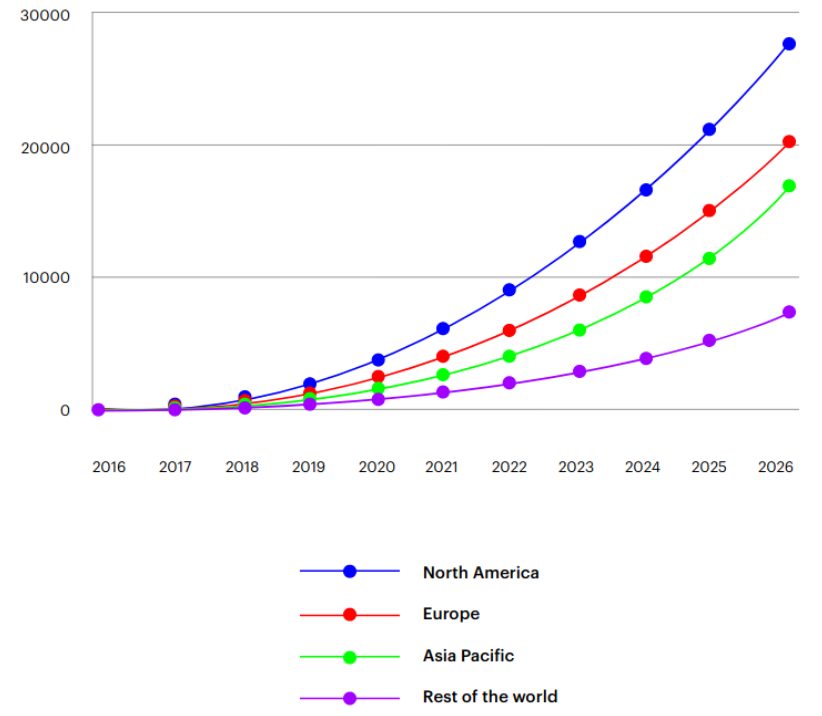

Fig.1

\section{PROPOSED WORK}

UML DIAGRAM

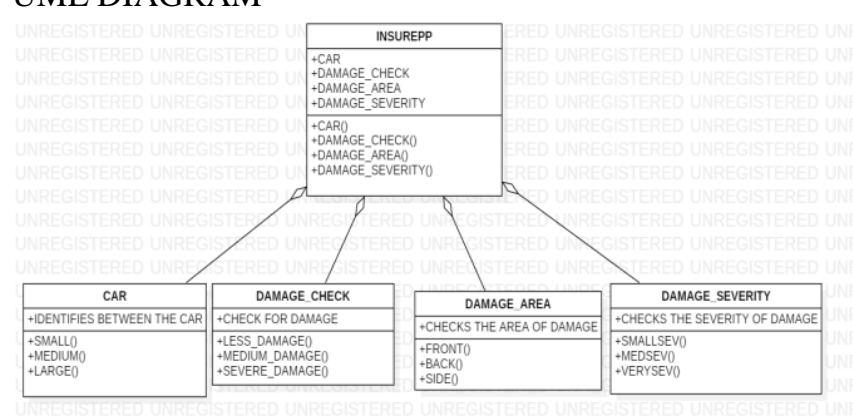

\section{HTML}

If we make some websites, then we require HTML.

It is mainly the skeleton of the websites which mainly consists of HTML,CSS,Javascript.It is mainly the building block of a particular website.The images,and other types of objects are mainly added by HTML.It mainly uses tags to function.

\section{CSS}

It helps HTML to make a website more attractive.It acts as skin of the website,as it helps in changing colours and other properties of a website.It's specification are mainly given in World Wide Web Consortium(W3).There are 3 types of CSS:internal,external and inline.

\section{VGG-16}

It is a CNN architecture and is considered one of the best models till date.It was used as an alternative over AlexNet as multiple $3 \times 3$ sized filters replaced some of the large sized kernel filters which was around 11 in first and 5 in the second convolutional layer.

\section{Grayscale}

Grayscaling is the way toward changing over a picture from other shading spaces e.g RGB, CMYK, HSV, and so forth to shades of grey. It shifts between complete dark and complete white.Some of it's advantages are dimension reduction(RGB has three dimensions but grayscale has only one dimension),model complexity reduction(if RGB is having 300 input nodes,grayscale has 100 nodes only),and there are many algorithms that work with grayscale image only.

\section{Normalize}

Normalize is used to convert real values into 0 or 1 .Machines normally can't read the numeric values

that we provide and can read only boolean values which is in the form of 0 or 1.It makes the models more accurate so that it can predict the outputs more easily.

\section{Flask}

It's mainly a micro framework which is coded in Python.It does not require any kind of data abstraction layer,form validation,etc types of components.It usually consists of Pocoo projects,Werkzeug and Jinja2.Pinterest and Linkedin uses Flask.

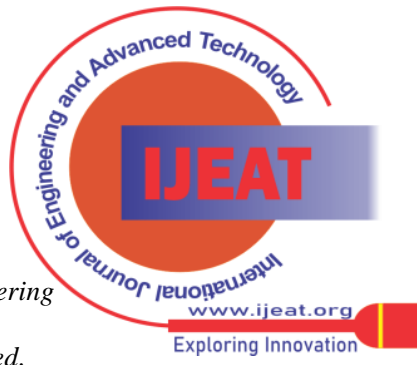




\section{Keras}

It is mainly used for running deep learning models. It mainly runs with tensorflow or Theano.It was developed so that deep learning models can work more fast and smoothly. The developer of Keras made it under 4 guidelines:modularity,minimalism,extensibility and Python.

\section{IMPLEMENTATION}

Using HTML CSS we created the frontend of our web page and connected it to python using Flask, the code contains 4 types of model - Car detection, Damage Detection, Side Detection and Severity Detection and then when we input the image using the web page it loads all the models and then one by one apply it on the image to create an output, by this we apply all 4 models and after this we created a weighted decision tree which decides the claim amount for the photo uploaded, by default if there is no damage on the car or no car then the default cost is Rs 50 .

We created the backend using machine learning to classify the image based on the pictures clicked to check whether the car is damaged or not.It uses VGG-16 for implementing the results.It mainly uses the formula ccclaim(cost,damage,location,severity)*1000.Here ccclaim() is a function which assigns a value according to the damage in the above 4 categories.

First of all,we will create a function ccclaim with parameters as cost,dmg,loc and sev.

Then we will develop a function grayscale so as to change the colour of picture from colourful to black and white.Then we will develop a function named normalize so as to convert the real values into 0 or 1.Then the 4 functions will be created namely

defisCar(),defisDamaged(),defdmgLoc(),defdmgSev().These functions are for check the conditions defined. To load the images, we

used

defloadIsCar(), defloadIsDamaged(), defloadDmgLoc(), deflo $\operatorname{adDmgSev}()$.

Then after this,we load all our models and it downloads all the data from tensorflow.After everything is done properly,it will print "All models loaded succesfully".

Then we can run our project.

\section{FIGURES AND TABLES}

TABLE I- We have made a table on 15 cars:

\begin{tabular}{|l|l|l|l|l|l|}
\hline S.NO & Cost & IsDmg & DmgLoc & Sev & $\begin{array}{l}\text { Total } \\
\text { Cost }\end{array}$ \\
\hline 1. & 1 & 1 & 1 & 1 & 9000 \\
\hline 2. & 0 & 0 & 2 & 0 & 50 \\
\hline 3. & 2 & 1 & 2 & 0 & 6000 \\
\hline 4. & 2 & 0 & 0 & 1 & 36000 \\
\hline 5. & 2 & 0 & 1 & 1 & 24000 \\
\hline 6. & 1 & 1 & 2 & 0 & 2250 \\
\hline 7. & 1 & 1 & 0 & 0 & 2250 \\
\hline 8. & 1 & 1 & 2 & 0 & 2250 \\
\hline 9. & 1 & 1 & 1 & 2 & 21000 \\
\hline 10. & 1 & 0 & 1 & 1 & 9000 \\
\hline 11. & 1 & 1 & 1 & 0 & 1500 \\
\hline 12. & 1 & 1 & 1 & 0 & 1500 \\
\hline 13. & 1 & 0 & 2 & 1 & 13500 \\
\hline 14. & 1 & 0 & 1 & 1 & 9000 \\
\hline 15. & 1 & 0 & 2 & 1 & 13500 \\
\hline & & & \multicolumn{2}{l}{} \\
\hline
\end{tabular}

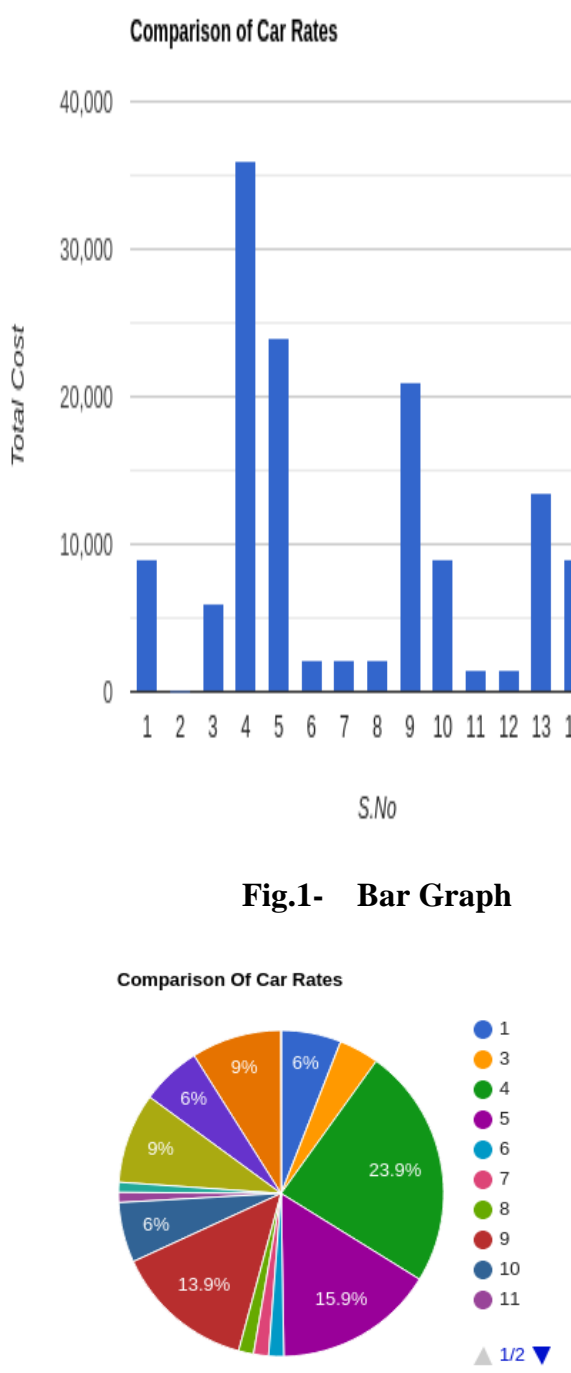

Fig.2- Pie Chart

VII. RESULT AND DISCUSSION

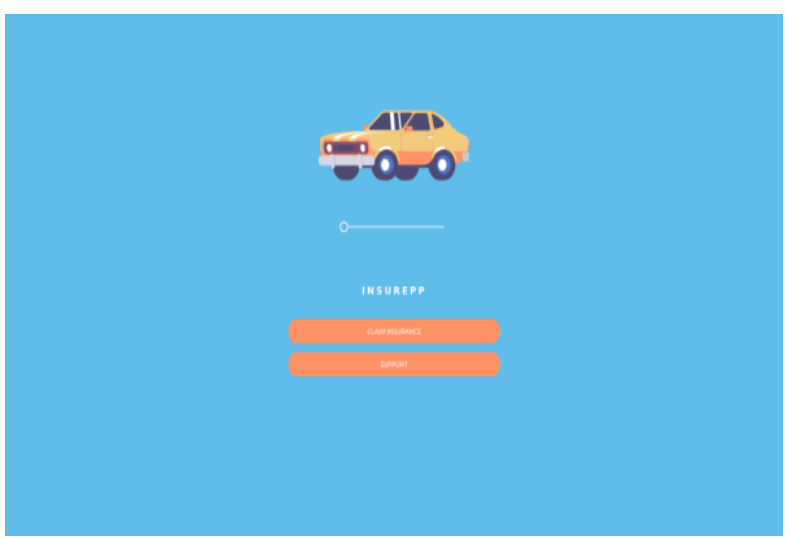

Fig.1-Main Page

Published By: Blue Eyes Intelligence Engineering and Sciences Publication (C) Copyright: All rights reserved. 


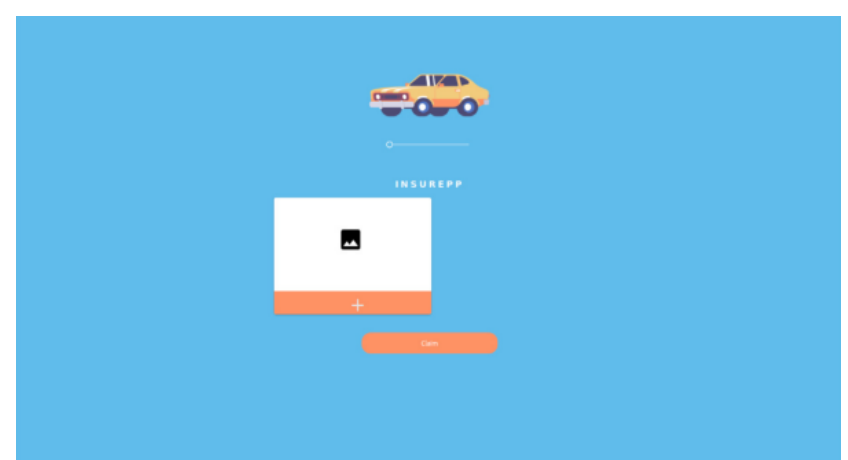

Fig.2-Page for uploading picture

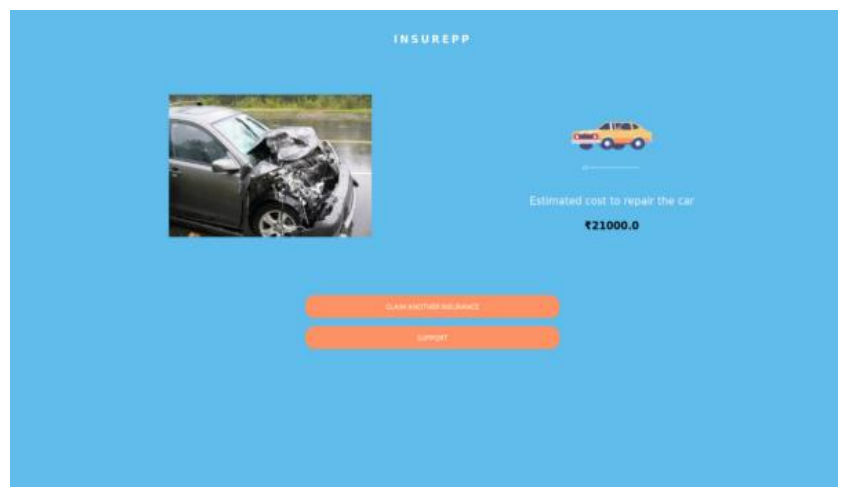

Fig.3-Result page

\section{ACKNOWLEDGMENT}

We want to thank our college SRM INSTITUTE OF SCIENCE AND TECHNOLOGY for giving us an opportunity to work on this project.It helped us to learn a lot of things which can be used in future works.It was a best opportunity for us also to learn something useful that would be required in future.

\section{REFERENCES}

1. Chien-Yao Wang (IIS Sinica), Hong-Yuan Mark Liao (IIS Sinica), I-Hau Yeh (Elan Microelectronics), Yueh-Hua Wu* (IIS Sinica), Ping-Yang Chen (NCTU), Jun-Wei Hsieh (NCTU):CSPNet: A New Backbone that can Enhance Learning Capability of CNN

2. Ehsan Hoseinzade and Saman Haratizadeh: CNNpred: CNN-based stock market prediction using a diverse set of variables

3. Jonathan Frankle and Michael Carbin:The Lottery Ticket Hypothesis: Finding Sparse, Trainable Neural Networks

4. Jayant D. Bokefode and Dr G. Komarasamy:License plate recognition using fast RCNN and OCR: NEED, TREND, CHALLENGES AND OPPORTUNITIES

5. Dr. Jennifer S. Raj:A NOVEL INFORMATION PROCESSING IN IOT

BASED

REAL TIME CNN photo Model and its SYSTEM

6. Pravin K shirsagar, Akshay Pote K., K. Paliwal, Vaibhav Hendre, Pranav Chippalkatti,Nikhil Dhabekar :Neural Network-Based Vehicle and Pedestrian Detection for Video

7. Dr. Sarika Jain, Anjali Grover, Praveen Singh Thakur, Sourabh Kumar Choudhary:Trends, Problems And Solutions of Recommender System

8. JIZHOU HUANG,HAIFENG WANG and WEI ZHANG:Multi-Task Learning for Entity Recommendation and Document Ranking in Web Search

9. Kaiming He, Georgia Gkioxari, Piotr Dollár, and Ross Girshick : Mask R-CNN

10. Yassine Ghouzam:Introduction to CNN Keras -0.997

11. https://www.accenture.com/ acnmedia/pdf-84/accenture-machine-le aning-insurance.pdf

\section{AUTHORS PROFILE}

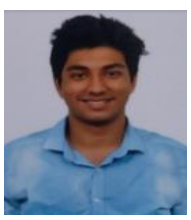

Rahul Singh, Student, Department of Computer Science and Engineering, SRM Institute of Science and Technology, Chennai (Tamil Nadu), India. Email: rr4872@srmist.edu.in

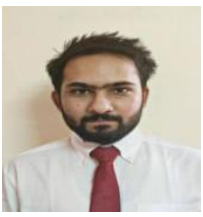

Avnesh Nigam, Student, Department of Computer Science and Engineering, SRM Institute of Science And Technology, Chennai (Tamil Nadu), India. Email: am3010@srmist.edu.in

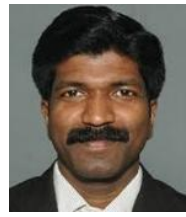

Dr S. Godfrey Winster, Assistant Professor, Department of Computer Science and Engineering, SRM Institute of Science And Technology, Chennai (Tamil Nadu), India. Email: godfreys@srmist.edu.in

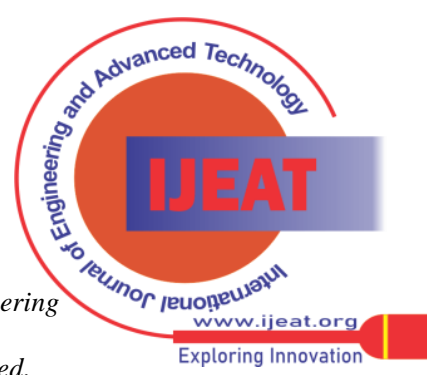

